\title{
Optimizing free parameters in the D3Q19 Multiple-Relaxation lattice Boltzmann methods to simulate under-resolved turbulent flows
}

\author{
M. Chávez-Modena ${ }^{a, b}$, A. Martínez-Cava ${ }^{a, b}$ G.Rubio $^{a, b}$ and E.Ferrer ${ }^{a, b}$ \\ ${ }^{a}$ ETSIAE - Universidad Politécnica de Madrid - Plaza de Cardenal Cisneros 3, 28040 Madrid, Spain \\ ${ }^{b}$ Center for Computational Simulation - Universidad Politécnica de Madrid, Campus de Montegancedo, \\ Boadilla del Monte, 28660 Madrid, Spain \\ m.chavez@upm.es
}

\begin{abstract}
We present a D3Q19 lattice scheme based in MRT with central moments (MRT$\mathrm{CM}$ ), where the free parameters of the model are optimized to dissipate high wavenumber under-resolved flow structures. In [16], the authors compared the BGK, MRT-RM and MRT-CM for the D2Q9 lattice scheme, using von Neumann analyses, to quantify their numerical properties, and based on these findings, proposed an optimized 2D MRT-CM scheme with enhanced stability for underresolved flows. Here, we extend the idea to the D3Q19 MRT-CM scheme. As before, we base our optimization, for the free parameters, on the $k-1 \%$ dispersion-error rule, that states that waves with dispersive errors above $1 \%$ should be dissipated since they pollute the solution and may cause instabilities. To this aim we increase dissipation in the scheme for waves with dispersive errors above $1 \%$.

The resulting optimized scheme is verified through a Von Newmann analysis and validated for the 3D Taylor-Green isotropic turbulent flow. We show how the original D3Q19 MRT-CM (d'Humières version) leads to unrealistic kinetic energy accumulation at high wave numbers, whilst our optimized MRT-CM provides the correct energy dissipated rate, avoiding energy build up at high wavenumbers. These results suggest that our optimization strategy enhances
\end{abstract}


stability and allows for accurate energy spectra in under-resolved flows simulations such as typically found in Large Eddy Simulations.

Keywords: Lattice Boltzmann, D3Q19 multiple-relaxation time, von Neumann analysis, rule of $\mathrm{k}-1 \%$ dispersion-error, homogeneous isotropic turbulence.

\section{Introduction}

Over the last decades, the Lattice Boltzmann method (LBM) has become an alternative to traditional discretization techniques of the Navier-Stokes (NS) equations to simulate fluid flows. Thanks to the efficient parallelization [1], the

5 LBM has a high computing performance being competitive against NS approaches for many applications. The LBM is capable of simulating situations where sound and flow interact, such as aeroacoustic generation [2, 3]. Additionally, it is to simulate a wide range of multiphase [4, 5] and multicomponent [6] flows. Despite its multiple use, in particular the turbulent flow simulations have to be validated properly because it appears in many applications. In this work, the homogeneous isotropic turbulence is computed to analyzed the turbulence quantities for different LB approaches.

The collision operator is responsibility of modeling the physics correctly, and has an strong effect in the numerical stability of the scheme [7, 8]. Several collision operators have been proposed to extend the range of applicability of the LBM. Recently, Coreixas et al. [9] proposed a formalism that encompasses all these approaches within a common mathematical framework. In this work, we focus into the single BGK and multiple-relaxation MRT time collision operators.

The most popular collision operator, the single-relaxation time, is based on the BGK [10] approximation. In this model a unique relaxation time is considered for all the probability distribution functions. As a result of its simplicity, it has severe stability limitations [11], precluding its use at low viscosities, large Mach numbers or under-resolved simulations.

On the other hand, the multiple-relaxation time with raw moments (MRTRM) collision operator [12] was introduced in an attempt to improve the BGK 
stability limitations. This operator enables different relaxation times for each probability distribution function. The increased complexity with respect to BGK resulted in a improved numerical stability [13].

Despite the enhancement in the stability of the MRT-RM with respect to the BGK collision operator, the MRT-RM can still show instabilities for small fluid viscosities [14]. Consequently, the MRT with central moments (MRTCM) collision operator was introduced [15]. Unlike the MRT-RM, where the moments are calculated in the global reference frame; the MRT-CM is calculated with the local macroscopic velocity. Using this formulation for the collision operator in the space of moments, it is possible to obtain a higher numerical stability because the higher dissipation at high wavenumbers [16].

Von Neumann stability analyses [17] enable the quantification of numerical errors in numerical schemes. Sterling and Chen [18] were the first to apply this analysis to the LB BGK approach. Then, Lallemand and Luo [13] used 40 it to compare the enhanced stability of the MRT-RM over the BGK approach. Subsequently, Siebert et al. [19] included high order terms into the equilibrium distribution of the D2Q9 model to improve the linear stability of the scheme (as shown depicted Fig. 2 in [19]). Malaspinas [20] proposed a new version of the BGK with improved stability and based on recursive relations and regularization

45 for the LB posed as Hermite series, which has been subsequently validated by Mattila et al. [21] and Coreixas et al. [22]. Later, in a previous work [16], we shown how the MRT-CM is more dissipative at higher wavenumbers compared with BGK and MRT-RM, which provides a better numerical stability.

Besides providing insight into numerical stability, this technique has been successfully used to provide insight into dispersive and dissipative errors. Marié et al. [23] compared BGK and MRT-RM collision operators. Dubois et al. [24] studied the numerical stability of the relative velocity (MRT-RM and MRT-CM) D2Q9 schemes with two different set of moments, proposed by Lallemand and Luo [13] and Geier et al. [14]. They concluded that MRT-CM with Geier's conservation law [14] had better stability properties. Recently, Gauthier et al. analyzed the information carried by the interaction between modes [25], giving 
an understanding to the reason of the numerical instability.

As mentioned, both the MRT-RM and the MRT-CM relax each hydrodynamic moment with a different relaxation time but all combinations lead to the same macroscopic state through the Chapman-Enskog expansion [26]. The relaxation times that are not fixed by the physics of interest become free parameters that can be optimized to enhance particular numerical aspects. Lallemand and Luo [13] optimized these parameters (for the D2Q9 lattice scheme) maximizing the Galilean invariance of the scheme, while reducing numerical errors (i.e. dispersion and dissipation). Similarly, $\mathrm{Xu}$ and Sagaut [2, 27] proposed an optimization to minimize dispersion/dissipation errors for the MRT-RM in D2Q9 scheme. Recently, in a previous work [16], we optimize the D2Q9 MRT-CM to increase dissipation only for high under-resolved wavenumbers (above the k-1\% dispersion-error), leaving low wavenumbers (well resolved scales) unchanged. This was tested successfully in the double periodic shear layer test.

In this work we extend previous ideas to improve the MRT-CM for 3D flows. The optimization strategy used previously for D2Q9[16] is now extended to the D3Q19 lattice scheme. Instead of minimizing dissipation errors for all wavenumbers, we propose to maintain numerical dissipation for well resolved wavenumbers whilst increasing dissipation for under-resolved wavenumbers. The optimization is inspired in the rule of $\mathrm{k}-1 \%$ dispersion-error presented by Moura et al. [28] in the context of high order numerical methods. They suggested that waves are only accurately resolved if the dispersion error (difference between theoretical and numerical) is below $1 \%$. The wavenumber at which the error becomes $1 \%$ was named "k-1\% dispersion-error" and lead to the " $1 \%$ rule". Following this rule, all waves above the k-1\% should be dissipated since these are poorly resolved and may pollute the solution. We follow the idea of damping under-resolved waves and apply it to the LBM for the first time in a 3D lattice scheme.

To assess the D3Q19 optimized MRT-CM, we simulate the Taylor-Green vortex (TGV) case [29] that includes starting transitional flow followed by decaying homogeneous turbulence. This case enalbes the quatification of vortex stretching/pairing 
processes and production of small-scale eddies, allowing the study of the dynamics of transition to turbulenc ans subsequent decaye. This test-case has been widely used to study dissipation errors of numerical schemes, of high order type, see e.g. [30, 40] and also LB schemes, e.g. [31, 32, 33].

The remaining of this text is organized as follows. First, in Section 2, we describe the numerical methodology which is divided in two parts: first, the Lattice Boltzmann method with the different collision operators and second the optimization strategy. Then, in Section 3, the results of the optimized approach are tested for the turbulent Taylor Green Vortex case. Finally, in Section 4 conclusions are presented.

\section{Methodology}

In this section the numerical methodology used in this work is presented. First, the Lattice Boltzmann method (LBM) is introduced. Special attention is paid to the definition of the collision operator. Secondly, an optimization method based on linear stability analysis is shown. The optimization aims at maximizing the robustness of the scheme for under-resolver simulations without penalizing its accuracy. The final objective is to improve the performance of the scheme for turbulent flows, therefore a three dimensional LBM scheme, in particular the D3Q19, is considered.

\subsection{Lattice Boltzmann method}

\subsubsection{Generalities}

The LBM is a numerical technique that provides numerical solutions of the continuous Boltzmann equation. The discrete Boltzmann equation reads:

$$
f_{i}\left(\mathbf{x}+\mathbf{e}_{i} \Delta t, t+\Delta t\right)-f_{i}(\mathbf{x}, t)=\Delta t \Omega_{i}(\mathbf{x}, t), i=0, \ldots, Q-1 .
$$

The discrete set of velocities is a vector of $Q$ components represented by the symbol $\mathbf{e}_{i}$. As a consequence, the discrete probability distribution functions (PDFs), $f_{i}\left(\mathbf{x}, \mathbf{e}_{i}, t\right)$, are stored at each lattice node for each time step, $\Delta t$. At 
each time step, the information stored in the discrete PDFs is streamed through the lattice and collided. The discrete collision operator $\Omega_{i}$ is the responsible of computing the post-collision state conserving mass and linear momentum.

The collision operator is of critical importance in the LBM method, as it is responsible of the modelization of the physics. The first approach for the collision operator was proposed by McNamara [34], but it was still rather complicated. The high cost of its evaluation precluded its use until Higuera and Jimenez [8] simplified this operator by performing linearization (under the assumption that the discrete PDF, $f_{i}$, is close to its equilibrium state). Expanding the discrete collision operator, $\Omega_{i}$, around the equilibrium state of the discrete PDF, $f_{i}^{e q}$, leads to a linearized operator:

$$
\Omega_{j}=K_{j i}\left(f_{i}^{\mathrm{eq}}-f_{i}\right),
$$

where $K_{j i}$ is known as collision matrix, and was further simplified to obtain the different collision operators. The collision matrix describes how the PDFs relax towards the equilibrium state. It is directly related with the viscosity and Mach number.

The discrete local equilibrium function $f_{i}^{\mathrm{eq}}$ is usually computed as a second order Taylor expansion of the Maxwell-Boltzmann distribution,

$$
f_{i}^{\mathrm{eq}}=\rho w_{i}\left(1+\frac{\mathbf{e}_{\mathbf{i}} \mathbf{u}}{c_{s}^{2}}+\frac{\left(\mathbf{e}_{\mathbf{i}} \mathbf{u}\right)^{2}}{2 c_{s}^{4}}-\frac{\mathbf{u}^{2}}{2 c_{s}^{2}}\right),
$$

where $w_{i}$ are weighting constants built to preserve isotropy and $c_{s}$ is the speed of sound. The particular values of the weighting constants $w_{i}$, depend on the discrete set of velocities [35].

\subsubsection{Collision operators}

\section{Single-relaxation time}

The single-relaxation time based on the Bhatnagar-Gross-Krook (BGK) [10] approximation is the most popular approach for the collision operator. In this case, using $K_{j i}^{\mathrm{BGK}}=(1 / \tau) \delta_{i j}$ (assuming $\delta_{i j}$ Kronecker delta notation) the collision 
operator simplifies to:

$$
\Omega_{i}^{\mathrm{BGK}}=\frac{1}{\tau}\left(f_{i}^{\mathrm{eq}}-f_{i}\right) .
$$

Only one relaxation time for all PDFs, $\tau$, is considered. The ChapmanEnskog expansion applied to the classical LBM equation (Eq. 1), with Eq. 4 as the discrete collision operator, establishes the relation between the relaxation time, the kinematic shear, $\nu$, and bulk viscosities, $\eta$, of the macroscopic fluid [36]:

$$
\nu=c_{s}^{2}\left(\tau-\frac{\Delta t}{2}\right), \quad \eta=\frac{2}{D} \nu,
$$

where $D$ is the dimension space. The BGK collision operator, is still widely used but has several shortcomings. For example, the kinematic viscosity is conditioned by the relaxation parameter, $\tau$, (see Eq. 5) which causes numerical instabilities for values near $\tau=0.5$ (with $\Delta t=1$ ), therefore complicating the simulation of high Reynolds number flows [11]. It should also be noticed that the bulk viscosity, $\eta$, cannot be freely chosen because it is constrained by the kinematic viscosity, $\nu$.

\section{Multiple-relaxation time}

In the MRT, the relaxation matrix is computed as a product of three matrices, $K_{j i}^{\mathrm{MRT}}=M_{j k}^{-1} S_{k l} M_{l i}$. The matrix $M$ accounts for the definition of the moments while the matrix $S$ is defined as a diagonal matrix:

$$
S_{k l}=s_{k} \delta_{k l},
$$

where $\mathbf{s}$ is a vector with relaxation times for the different moments. The MRT$\mathrm{CM}$ collision operator is obtained defining the collision matrix as $K_{j i}^{\mathrm{MRT}-\mathrm{CM}}=$ $M_{j k}(\mathbf{u})^{-1} S_{k l} M_{l i}(\mathbf{u})$ :

$$
\Omega_{j}^{\mathrm{MRT}-\mathrm{CM}}=M_{j k}(\mathbf{u})^{-1} S_{k l} M_{l i}(\mathbf{u})\left(f_{i}^{\mathrm{eq}}-f_{i}\right) .
$$

This notation allows working with the equilibrium PDF, $f_{i}^{\text {eq }}$ defined in the BGK model. Note that Eq. 7 corresponds the MRT-CM because it takes into account the macroscopic velocity, $\mathbf{u}$. On the other hand, if $\mathbf{u}=\mathbf{0}$ the MRT with 
raw moments (MRT-RM) is obtained. Additionally, note that the BGK collision operator can be recovered by setting all the diagonal elements $S_{i i}$ to $1 / \tau$.

The formulation introduced up to this point is general for any lattice distribution and spatial dimensions. In this work we aim at improving the performance of LBM for under-resolved turbulent flows, therefore a three dimensional LBM is considered. In particular we focus on the popular D3Q19, whose moment matrix, $M$, can be found in Appendix A

\subsection{Optimization method for the MRT-CM}

In this section the optimization method to improve the performance of LBM for turbulent flows is introduced. This method, introduced by the authors in a previous work [16], modifies the relaxation times of the collision operator in order to maximize the dissipation of under-resolved wavelengths. The optimization method makes use of linear stability analysis to separate well-resolved and under-resolved wavelengths.

\subsubsection{Linear stability analysis}

\section{Theoretical modes}

The theoretical modes are obtained through an analytic linearization of the NS equations [37]. The resulting hydrodynamic modes are known as shear mode, $\omega_{t}^{s}$, and acoustic modes, $\omega_{t}^{ \pm}$. The first is related to the kinematic shear viscosity. The second is related to both kinematic shear and bulk viscosities. The analytical expressions for the theoretical hydrodynamic modes read:

$$
\begin{aligned}
& \omega_{t}^{s}=\mathbf{u} \cdot \mathbf{k}-\mathrm{i} \nu|\mathbf{k}|^{2}, \\
& \omega_{t}^{ \pm}=\left(\mathbf{u} \pm c_{s}\right) \cdot \mathbf{k}-\mathrm{i}\left[\frac{(D-1)}{D} \nu+\frac{\eta}{2}\right],
\end{aligned}
$$

where $\mathbf{k}$ is the wavenumber. For an illustrative purpose Figure 1 depicts dispersion (real part of $\omega, \operatorname{Re}(\omega)$ ) and dissipation (imaginary part of $\omega, \operatorname{Im}(\omega)$ ) behavior for a range of wavenumbers $[0, \pi]$, following Eq. 8 for three-dimensions $(D=3)$, 


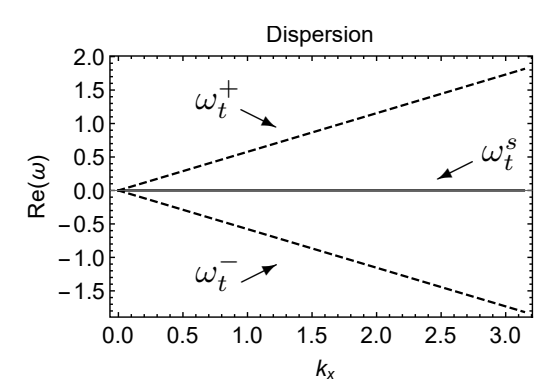

(a)

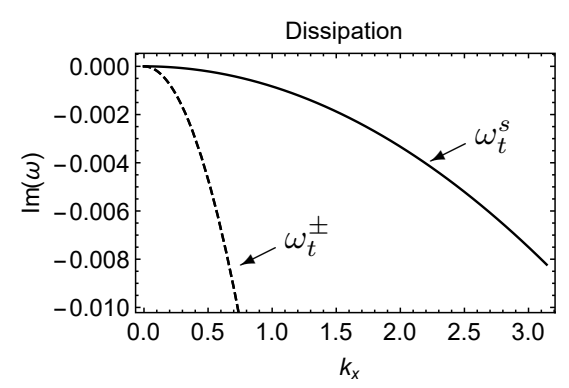

(b)

Figure 1: Dispersion, $\operatorname{Re}(\omega)$, (a) and dissipation, $\operatorname{Im}(\omega)$, (b) of the three-dimension theoretical shear mode, $\omega_{t}^{s}$ : - , and acoustic modes, $\omega_{t}^{ \pm}: \quad \mathbf{- -}$, at Ma $=0$ with $\nu=10^{-3} \frac{\mathrm{m}^{2}}{\mathrm{~s}}$ and $\eta=3.66 \cdot 10^{-2} \frac{\mathrm{m}^{2}}{\mathrm{~s}}$.

$\mathbf{u}=\left\{u_{x}, 0,0\right\}$ and $\mathbf{k}=\left\{k_{x}, 0,0\right\}$.

170

\section{Numerical modes}

The numerical modes can be obtained by means of von Neumann (VN) analysis [18]. Von Neumann analysis splits the PDF into an equilibrium state, $\bar{f}_{i}$, plus a perturbation, $\delta f_{i}(x, t)$ :

$$
f_{i}(x, t)=\bar{f}_{i}+\delta f_{i}(\mathbf{x}, t) .
$$

The first term in the right hand side, $\bar{f}_{i}$, is the global PDF, which does not vary with time and space i.e., it depends only on the average density and velocity. The second term in the right hand side, $\delta f_{i}(x, t)$, accounts for the fluctuations from equilibrium. The fluctuation is assumed as a sinusoidal wave $F_{i} e^{i(\mathbf{k x}-\omega t)}$ with an amplitude $F_{i}$.

Substituting Eq. 9 into Eq. 2, expanding the $f_{i}^{e q}$ by means of Taylor series centred at the global distribution and after rearranging the expression, an eigenvalue problem is obtained, $F_{i} \lambda=F_{j} G_{i j}$, where $\lambda=e^{-i \omega \Delta t}$ are the eigenvalues of the amplification matrix, $G_{i j}$, defined as:

$$
G_{i j}=A_{i k}^{-1}\left[\delta_{k j}+\Delta t K_{k n} N_{n j}\right] \text { with }\left\{\begin{array}{l}
A_{i k}=\delta_{i k} e^{i \mathbf{k} \Delta t \mathbf{e}_{\mathbf{i}}}, \\
N_{n j}=\frac{\partial f_{n}^{e q}}{\partial f_{j}}-\delta_{n j},
\end{array}\right.
$$


where the different collision matrices, $K_{i j}$, are defined in Section 2.1.2 For more details on the VN analysis for the LBM, see [18, 16].

\subsubsection{Optimization process: Rule of $k-1 \%$ dispersion-error}

The optimization described here has been inspired by the rule of $\mathrm{k}-1 \%$ dispersionerror proposed by Moura et al. [28] in the context of hp spectral methods [38, 39, 40]. Moura et al. suggested that wavenumbers with dispersion errors (difference between theoretical and numerical dispersion modes) higher than $1 \%$ should be dissipated, since high dispersion errors tend to pollute the solution. In other words, a high dissipative error at high wavenumbers is a positive feature of a numerical method. In a previous work [16], we proposed to optimize the values of the free relaxation times (those affecting moments of order higher than two), to maximize the dissipation of the shear mode in wavenumbers higher than the k-1\% dispersion-error wavenumber for the D2Q9 MRT-CM. The dissipation level is measured as the area defined by the theoretical shear mode and numerical shear mode curves for wavenumbers higher than $\mathrm{k}-1 \%$ dispersion-error wavenumber (green area in Figure 2). The error for the k-1\% dispersion-error is calculated with the shear mode (theoretical and numerical) by means of the following expression:

$$
\% \operatorname{Error}(\mathbf{k})=\frac{\left|\omega_{s}-\omega_{s}^{t}\right|}{\omega_{s}^{t}} \cdot 100 .
$$

As the rule describes, $\mathrm{k}-1 \%$ dispersion-error wavenumber occurs when this error is $1 \%$. The dissipation of acoustic modes has not been taken into account because they barely change with the free parameters.

\subsubsection{Optimized MRT-CM}

This section details the optimization process for D3Q19 MRT-CM lattice scheme. The optimization aims at maximizing the dissipation of the shear mode in wavenumbers higher than the $\mathrm{k}-1 \%$ dispersion-error wavenumber by modifying the values of the free relaxation times $\left(s_{10-18}\right)$. 


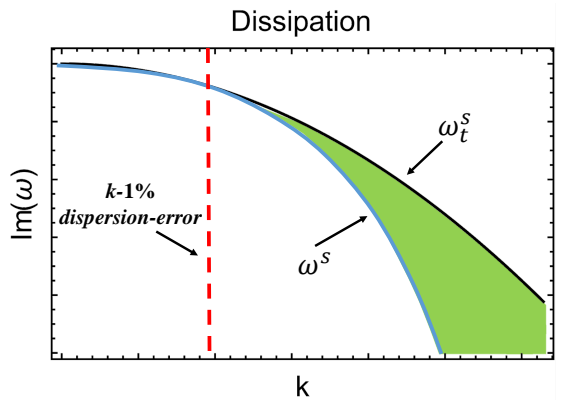

Figure 2: Dissipation area calculated from the difference between the theoretical, $\omega_{s}^{t}$, and numerical, $\omega_{s}$, shear mode.

The free parameters $s_{10,11,12}, s_{13,14,15}$ and $s_{16,17,18}$ are grouped due to moments symmetry constrains. Besides, only the first two groups are considered in the optimization, as we found that the last group does not affect the shear dissipation mode. As a result, $s_{16,17,18}$ has been fixed to 1.4, the value proposed by d'Humieres [41]. The flow direction and the perturbations are considered parallel to the lattice direction in the optimization process, i.e., $\mathbf{u}=\left\{u_{x}, 0,0\right\}$ and $\mathbf{k}=\left\{k_{x}, 0,0\right\}$. As numerical and theoretical modes depend on the fluid conditions (Mach number, Ma and viscosity $\nu$ ), the free relaxation times of the model have been optimized for different values of Mach number (Ma $=0.1,0.2$ and 0.3$)$ and viscosity $\left(\nu=\eta=10^{-3}\right.$, $10^{-4}$ and $10^{-5}$ ).

The optimal values of $s_{10,11,12}$ and $s_{13,14,15}$ are found by a brute force approach where the parametric space of the free parameters is discretized with $\Delta s_{i}=$ 0.01 (i.e. a tolerance of $1 \%$ ). Besides, only values of the relaxation times in the range $[0,2[$ are considered. Table 1$]$ shows the optimal values of these parameters. It can be observed that the values are dependent on Ma and are almost independent of the viscosity (see Table 1). These values are very different from the free parameters proposed by d'Humières [41] were $s_{10-12}=$ $1.98, s_{13-15}=1.98$ and $s_{16-18}=1.4$.

To illustrate the effect of the optimization, the numerical modes of the optimized MRT-CM are compared with the ones of d'Humières' MRT-CM in 


\begin{tabular}{c|cc|cc|cc} 
& \multicolumn{2}{|c}{ Ma $=0.1$} & \multicolumn{2}{c}{ Ma $=0.2$} & \multicolumn{2}{c}{ Ma $=0.3$} \\
$\nu=\eta$ & $s_{10-12}$ & $s_{13-15}$ & $s_{10-12}$ & $s_{13-15}$ & $s_{10-12}$ & $s_{13-15}$ \\
\hline $10^{-3}$ & 0.62 & 0.81 & 0.92 & 1.12 & 1.11 & 1.32 \\
$10^{-4}$ & 0.61 & 0.80 & 0.90 & 1.10 & 1.10 & 1.30 \\
$10^{-5}$ & 0.61 & 0.80 & 0.90 & 1.10 & 1.10 & 1.30
\end{tabular}

Table 1: Optimized values for $s_{10}=s_{11}=s_{12}$ and $s_{13}=s_{14}=s_{15}$ at different viscosities and Mach numbers.

Figure 3. These modes are computed for $\mathrm{Ma}=0.2, \nu=10^{-3} \frac{\mathrm{kg}}{\mathrm{ms}}$ and $\eta=$ $6.7 \cdot 10^{-4} \frac{\mathrm{kg}}{\mathrm{ms}}$. The values of the Mach number, Ma, viscosities, $\nu$ and $\eta$, and wavenumbers, $k$, have been taken from [16] to permit a comparison of the results. It can be seen that the optimized MRT-CM presents a higher dissipation rate at high wavenumbers than d'Humières MRT-CM. This should result in an enhanced robustness for under-resolved simulations. Besides, it should be noticed that this increase in the dissipation rate does not affect the dispersion error at low wavenumbers. As a result, similar behaviour of the optimized MRT-CM and d'Humières MRT-CM is expected for low wavenumbers. Both approaches will be tested for an isotropic turbulent flow in the following section.

In Appendix B we have included a comparison between the numerical modes of BGK, MRT-RM and MRT-CM for D3Q19 lattice scheme. Besides, some insight on the effect of the relaxation parameters on numerical modes is given in Appendix C where d'Humières [41] and Lallemand's [13] relaxation times are compared for D3Q19 lattice scheme.

\section{Numerical validation}

In this section the new optimized scheme is compared with previous approaches found in the literature. The aim of this section is to show that the proposed scheme provides increased stability without penalizing the accuracy.

In order to study the effect of the present optimized MRT-CM on a threedimensional turbulent configuration, the decaying Taylor-Green vortex (TGV) 


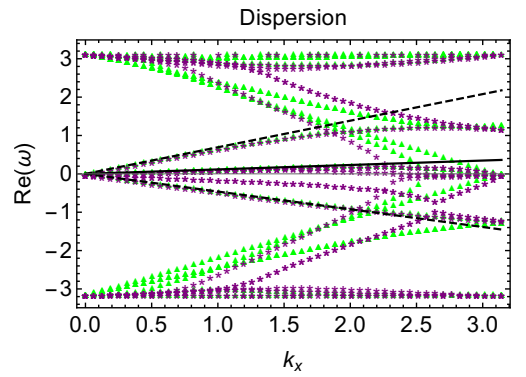

(a)

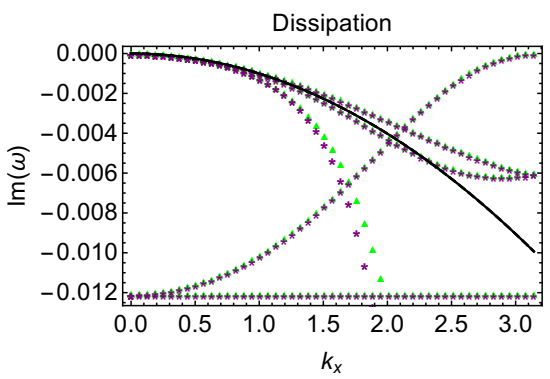

(b)

Figure 3: Dispersion (a) and dissipation (b) for D3Q19 with d'Humières' MRT-CM: $\Delta$, optimized MRT-CM: $*$ and theoretical modes (shear mode: - and acoustic modes: - - ) at Ma $=0.2$ with $\nu=10^{-3} \frac{\mathrm{kg}}{\mathrm{ms}}$ (Note that the dissipation theoretical modes are overlapped).

[29] has been simulated. It is a fundamental test case used as prototype for vortex stretching and production of small-scale eddies and therefore allows the study of the dynamics of transition to turbulence. This test-case has been widely used to study the dissipation errors of numerical schemes [30]. In the LB context, few studies have been validated their approaches with the threedimensional TGV [31, 32].

In this work, the TGV is simulated with BGK, d'Humières' MRT-CM and optimized MRT-CM with different lattice resolutions. Grid resolution of $N=$ $32,64,128$ and 256 have been computed, but the analysis has focused in the under-resolved resolutions ( $N=32$ and 64 ), to prove if the new optimized approach provides a correct dissipation evolution.

\subsection{Simulation setup}

The TGV problem can be run using a variety of flow and initial conditions. However, the conditions and post processing used here were specified by the organizers of the AIAA First International Workshop on High-Order Methods in Computational Fluid Dynamics [30].

The domain simulated consists of a cube with length defined as $-\pi L<$ $x, y, z<\pi L$. The boundary conditions are periodic and the initial conditions of 
the simulation are selected as:

$$
\begin{aligned}
& \mathbf{u}_{x}=U_{0} \sin [x / L] \cos [y / L] \cos [z / L], \\
& \mathbf{u}_{y}=-U_{0} \cos [x / L] \sin [y / L] \cos [z / L], \\
& \mathbf{u}_{z}=0, \\
& p_{0}=\frac{\rho_{0} U_{0}^{2}}{16}(\cos [2 x / L]+\cos [2 y / L])(\cos [2 z / L]+2),
\end{aligned}
$$

where the reference density and length, $\rho_{0}$ and $L$, are set to one.

The reference velocity, $U_{0}$, is selected through the Mach number, $\mathrm{Ma}=$ $U_{0} / c_{s}=0.1$. The kinematic shear viscosity is set through the relation with the Reynolds number, $\operatorname{Re}=U_{0} L / \nu=1600$. The physical time computed in each simulation is $t=20 t_{c}$, where the characteristic time, $t_{c}$, is defined as $t_{c}=L / U_{0}$. Besides, different lattice numbers $(N=32,64,128$ and 256) have been used in order to test the different collision operators.

\subsection{Results}

To analyze the capabilities of each collision operator the dynamics of a three-dimensional decaying vortex has been computed and compared with a DNS simulation performed with a spectral method [30]. In the following, the dissipation rate and the spectrum of the kinetic energy is scrutinized for various under-resolved grids.

\subsubsection{Reference results}

The TGV evolution is characterized by three main steps visible in the time trace of kinetic energy dissipation rate, $\varepsilon$ (see Eq. 13). First, the initial laminar state is transitioning to turbulence until the stretched vortex tubes break down into small scales around $t / t_{c}=5$. Then the dissipation rate rises to a sharp peak near $t / t_{c}=9$ corresponding to the fully turbulent state which is then decaying similarly to an isotropic and homogeneous turbulence. Figure 4 shows the different steps described through the time evolution of the kinetic energy dissipation rate, $\varepsilon$, for $\mathrm{Re}=1600$ and $\mathrm{Ma}=0.1$. Flow structures are represented by isosurface of the Q-criterion colored by kinetic energy. The simulation on a 
$N=256$ grid is in very good agreement with the spectral results also plotted in Figure 4.

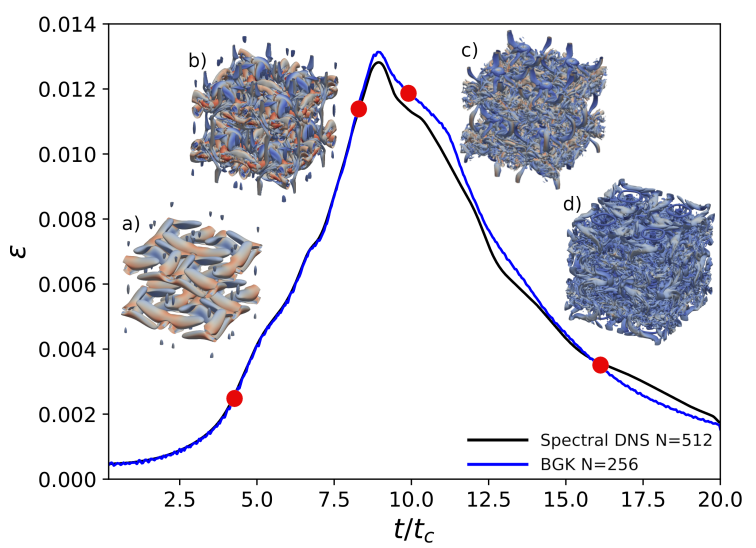

Figure 4: Isosurface of the Q-criterion colored by kinetic energy at time $t / t_{c}=4$ (a), 8 (b), 10 (c), 16 (d) for $\operatorname{Re}=1600$ on a $N=256$ grid compared with DNS results with $N=512$ [30].

\subsubsection{Kinetic energy dissipation rate}

As illustrated before, in order to study the evolution of the fluid the temporal evolution of the kinetic energy dissipation rate $\varepsilon$ is calculated:

$$
\varepsilon=-\frac{d E_{k}}{d t}
$$

As shown by Eq. 13, the kinetic energy dissipation rate is the temporal derivative of the kinetic energy, $E_{k}$, which is estimated using the following formula:

$$
E_{k}=\frac{1}{\rho_{0} V} \int \rho \frac{\mathbf{u}^{2}}{2} d V
$$

where the density is $\rho=\rho_{0}$ and $V$ is the volume of the domain.

The results obtained with this definition are compared to the kinetic energy dissipation rate of the reference [30]. Figure 5 shows the time evolution of the kinetic energy dissipation rate, $\varepsilon$, for BGK and MRT-CM (with d'Humières' and 

spectral DNS solution $N=512$.

Analyzing the BGK behavior, the $N=32$ and 64 simulations give rise to numerical instability at different times, while the $N=128$ grid is stable. The $N=32$ becomes unstable almost at the beginning of the simulation. The $N=64$ grid simulation collapses earlier around $t / t_{c}=5$, when the stretched vortex tubes break down into small scales. However, the $N=128$ grid gives satisfactory results for the transition region and the peak but the decaying phase is not properly captured.

Regarding the MRT-CM, both set of relaxation times are stable with the eddies contain most of the kinetic energy, whereas the smallest eddies are responsible for the viscous dissipation of kinetic energy.

Therefore, since turbulence exists with a range of eddy sizes, it is frequently 


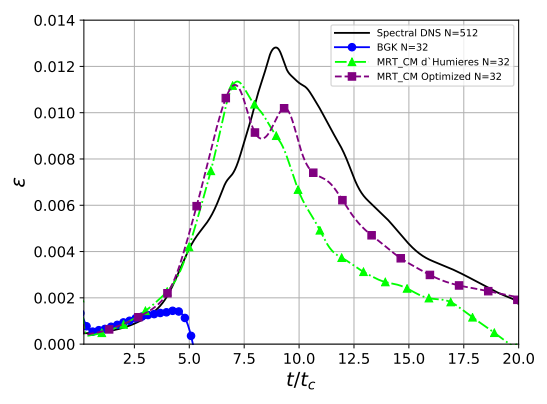

(a)

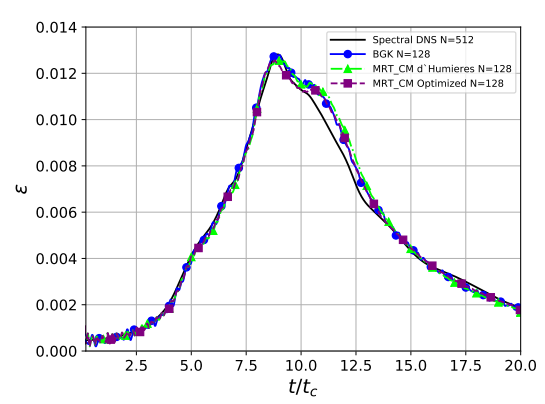

(c)

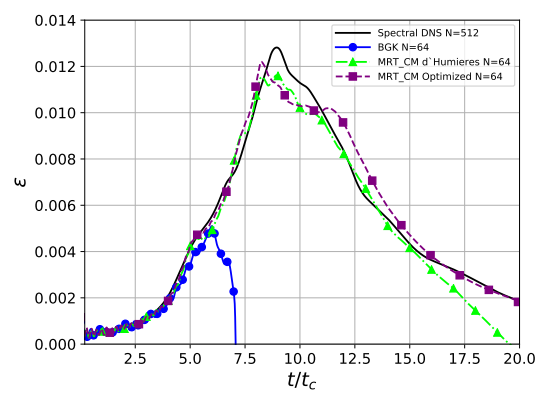

(b)

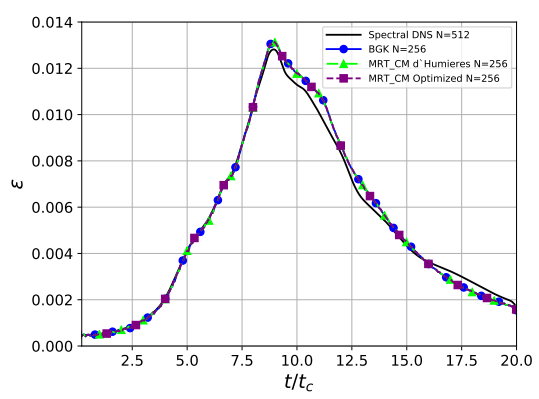

(d)

Figure 5: Kinetic energy dissipation rate for BGK: •, d'Humières' MRT-CM: $\Delta$, and optimized MRTCM: $\square$, on a grid $N=32$ (a), $N=64$ (b), $N=128$ (c) and $N=256$ (d) at $\operatorname{Re}=1600$ and Ma $=0.1$.

convenient to take the Fourier transform of the velocity field in order to consider the Fourier components of different wavenumbers. The components of the velocity in Fourier space will be:

$$
\mathbf{u}(\mathbf{k})=\int \mathbf{u}^{\prime}(\mathbf{x}) e^{-\mathbf{i k x}} d \mathbf{x}
$$

where $\mathbf{u}^{\prime}$ is the turbulent component velocity. Then, the energy spectrum function $E(\mathbf{k})$ is a characterization of the energy turbulent flow in the case of homogeneous turbulence obtained, it reads:

$$
E(\mathbf{k})=\frac{1}{2} \mathbf{u}^{\prime 2}
$$


Continuing with the analysis, in order to understand how the optimization affects to the turbulence dissipation at the different wavenumbers, Figure 6 shows the kinetic energy spectra of the optimized parameters compared with d'Humières' parameters at different grids and time steps. The Kolmogorov law in spectral form $E(k) \sim k^{-\frac{5}{3}}$ and the cut-off wavenumber, $k_{c}$, are used for the comparison. The cut-off wavenumber is the maximum wavenumber for resolved length scales.

Under-resolved resolutions ( $N=32$ and $N=64$ ) have been selected to test if the optimized approach provides a correct evolution tendency dissipating the high wavenumbers. First, notice how the differences between both set of parameters decrease when the grid resolution increases. Then, at $t / t_{c}=6$ and 8 , before and after the dissipation peak (see Figure 5) the optimized values are less dissipative enabling higher wavenumbers (smaller eddies). Moreover, d'Humières' MRT-CM has an extra kinetic energy accumulation at high wavenumbers. This results in a wrong decay of kinetic energy compared with the theoretical slope of $k^{-\frac{5}{3}}$. However, with our optimized MRT-CM, the energy is dissipated correctly obtaining a correct energy decay. These results suggest that the optimization strategy applied is able to solve correctly the dynamics of transition to turbulence, giving an adequate trend in energy decay for isotropic turbulence. 

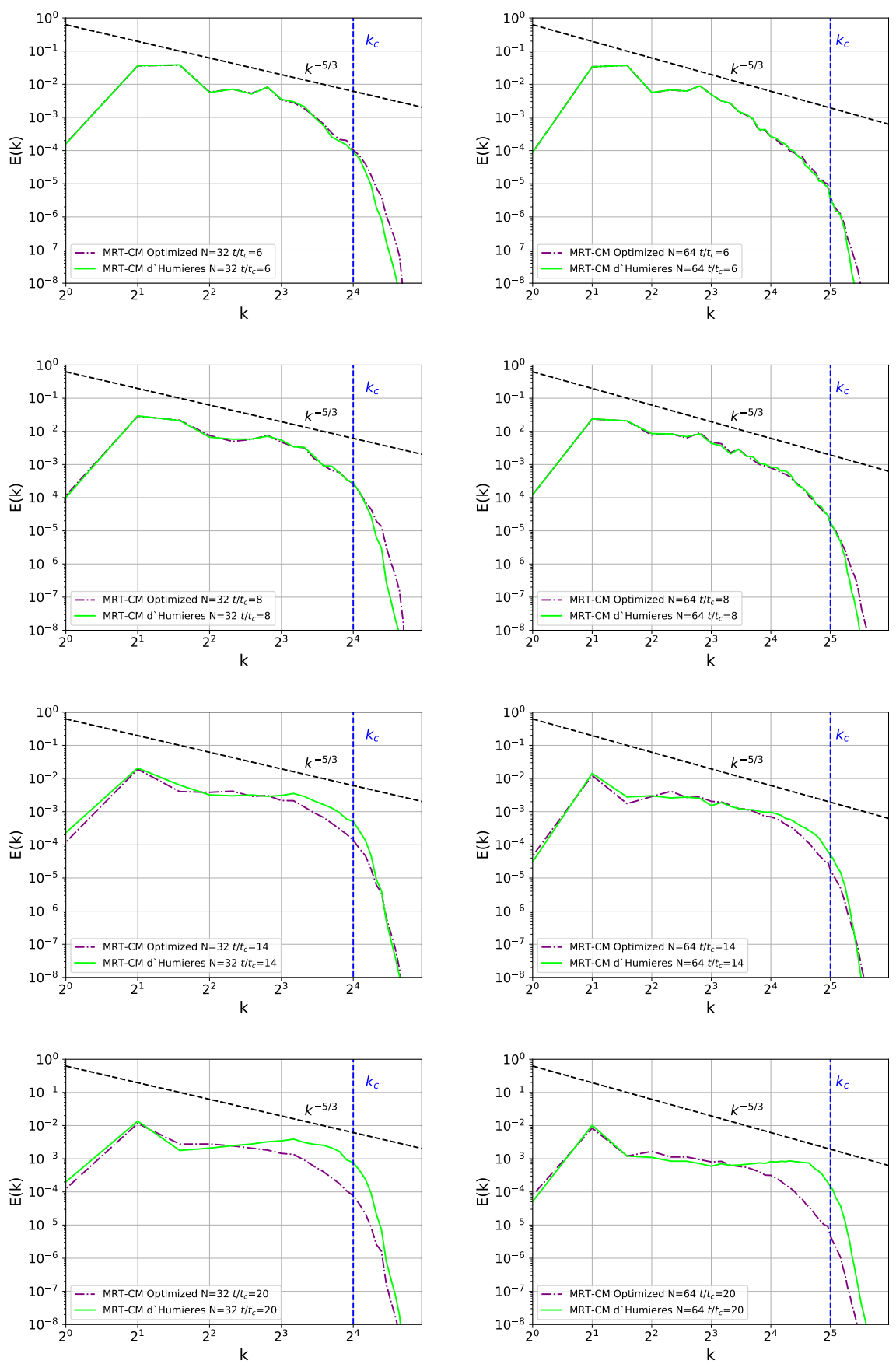

Figure 6: Kinetic energy spectra at $t / t_{c}=6,8,14,20$ for d'Humières' MRT-CM: $\quad$, and optimized MRT-CM: - on a $N=32$ (left) and $N=64$ (right) grid; $\mathrm{Re}=1600$ and Ma $=0.1\left(k^{-5 / 3}\right.$ : and $\left.k_{c}: \quad \mathbf{-}\right)$. 


\section{Acknowledgment}

This project has received funding from the European Unionś Horizon 2020

research and innovation programme under grant agreement No 785549 (FireExtintion: H2020-CS2-CFP06-2017-01).

The authors acknowledge the computer resources and technical assistance provided by the Centro de Supercomputación y Visualización de Madrid (CeSViMa). 
Based on Geier et al. [14] moments, the following set of moments is proposed:

$$
\begin{aligned}
X^{m} Y^{l} Z^{k}= & \left(X^{0} Y^{0}, X, Y, Z, X Y, X Z, Y Z, X^{2}-Y^{2}, X^{2}-Z^{2}, X^{2}+Y^{2}+Z^{2},\right. \\
& X Y^{2}+X Z^{2}, X^{2} Y+Y Z^{2}, X^{2} Z+Y^{2} Z, X Y^{2}-X Z^{2}, X^{2} Y-Y Z^{2}, X^{2} Z-Y^{2} Z, \\
& \left.X^{2} Y^{2}+X^{2} Z^{2}+Y^{2} Z^{2}, X^{2} Y^{2}+X^{2} Z^{2}-Y^{2} Z^{2}, X^{2} Y^{2}-X^{2} Z^{2}\right)^{T} ; m, l, k=0,1,2
\end{aligned}
$$

Applying the Gram-Schmidt orthogonalization procedure to Eq. A.1, the matrix $M(\mathbf{u})$ is obtained:

$$
M(\mathbf{u})=\left(\begin{array}{c}
X^{0} Y^{0} Z^{0} \\
X \\
Y \\
Z \\
X Y \\
X Z \\
Y Z \\
X^{2}-Y^{2} \\
\left(X^{2}+Y^{2}+Z^{2}\right)-3 Z^{2} \\
\left(X^{2}+Y^{2}+Z^{2}\right)-2 \\
3\left(X Y^{2}+X Z^{2}\right)-4 X \\
3\left(Y X^{2}+Y Z^{2}\right)-4 Y \\
3\left(Z X^{2}+Z Y^{2}\right)-4 Z \\
\left(X Y^{2}-X Z^{2}\right) \\
\left(Y X^{2}-Y Z^{2}\right) \\
\left(Z X^{2}-Z Y^{2}\right) \\
\left.j_{y}\right|_{i} \\
\left.j_{z}\right|_{i} \\
\left.p_{x y}\right|_{i} \\
\left.p_{x z}\right|_{i} \\
\left.p_{y z}\right|_{i} \\
\left.p_{w w}\right|_{i} \\
\left.3 p_{x x}\right|_{i} \\
\left.e\right|_{i} \\
\left.q_{x}\right|_{i} \\
\left.q_{y}\right|_{i} \\
\left.q_{z}\right|_{i} \\
\left.m_{x}\right|_{i} \\
\left.m_{y}\right|_{i} \\
\left.m_{z}\right|_{i} \\
\left.3 \pi_{x x}\right|_{i} \\
\left.\pi_{w w}\right|_{i} \\
\left.\epsilon\right|_{i}
\end{array}\right)
$$

As can be seen, the selection of Geier moments has a physical foundation. $\rho$ is the density, $e$ is the kinetic energy, $\epsilon$ is related to the kinetic energy square, $\mathbf{j}=\rho \mathbf{u}$ components correspond to components of momentum, $\mathbf{q}$ components 
correspond to the internal energy components, $\mathbf{p}$ components correspond to the symmetric traceless viscous stress tensorsm, $\pi$ is related with the kinetic energy and the viscous stress tensor and $m$ components are the asymmetric third-order moments.

Hence the transformation matrix in raw moments is $M(\mathbf{u}=\mathbf{0})$ with $X=e_{x}$, $Y=e_{y}$ and $Z=e_{z}$.

$$
M(\mathbf{0})=\left(\begin{array}{ccccccccccccccccccc}
1 & 1 & 1 & 1 & 1 & 1 & 1 & 1 & 1 & 1 & 1 & 1 & 1 & 1 & 1 & 1 & 1 & 1 & 1 \\
0 & -1 & 0 & 0 & -1 & -1 & -1 & -1 & 0 & 0 & 1 & 0 & 0 & 1 & 1 & 1 & 1 & 0 & 0 \\
0 & 0 & -1 & 0 & -1 & 1 & 0 & 0 & -1 & -1 & 0 & 1 & 0 & 1 & -1 & 0 & 0 & 1 & 1 \\
0 & 0 & 0 & -1 & 0 & 0 & -1 & 1 & -1 & 1 & 0 & 0 & 1 & 0 & 0 & 1 & -1 & 1 & -1 \\
0 & 0 & 0 & 0 & 1 & -1 & 0 & 0 & 0 & 0 & 0 & 0 & 0 & 1 & -1 & 0 & 0 & 0 & 0 \\
0 & 0 & 0 & 0 & 0 & 0 & 1 & -1 & 0 & 0 & 0 & 0 & 0 & 0 & 0 & 1 & -1 & 0 & 0 \\
0 & 0 & 0 & 0 & 0 & 0 & 0 & 0 & 1 & -1 & 0 & 0 & 0 & 0 & 0 & 0 & 0 & 1 & -1 \\
0 & 1 & -1 & 0 & 0 & 0 & 1 & 1 & -1 & -1 & 1 & -1 & 0 & 0 & 0 & 1 & 1 & -1 & -1 \\
0 & 1 & 1 & -2 & 2 & 2 & -1 & -1 & -1 & -1 & 1 & 1 & -2 & 2 & 2 & -1 & -1 & -1 & -1 \\
-2 & -1 & -1 & -1 & 0 & 0 & 0 & 0 & 0 & 0 & -1 & -1 & -1 & 0 & 0 & 0 & 0 & 0 & 0 \\
0 & 4 & 0 & 0 & 1 & 1 & 1 & 1 & 0 & 0 & -4 & 0 & 0 & -1 & -1 & -1 & -1 & 0 & 0 \\
0 & 0 & 4 & 0 & 1 & -1 & 0 & 0 & 1 & 1 & 0 & -4 & 0 & -1 & 1 & 0 & 0 & -1 & -1 \\
0 & 0 & 0 & 4 & 0 & 0 & 1 & -1 & 1 & -1 & 0 & 0 & -4 & 0 & 0 & -1 & 1 & -1 & 1 \\
0 & 0 & 0 & 0 & -1 & -1 & 1 & 1 & 0 & 0 & 0 & 0 & 0 & 1 & 1 & -1 & -1 & 0 & 0 \\
0 & 0 & 0 & 0 & -1 & 1 & 0 & 0 & 1 & 1 & 0 & 0 & 0 & 1 & -1 & 0 & 0 & -1 & -1 \\
0 & 0 & 0 & 0 & 0 & 0 & -1 & 1 & 1 & -1 & 0 & 0 & 0 & 0 & 0 & 1 & -1 & -1 & 1 \\
0 & -4 & 2 & 2 & 1 & 1 & 1 & 1 & -2 & -2 & -4 & 2 & 2 & 1 & 1 & 1 & 1 & -2 & -2 \\
0 & 0 & -2 & 2 & 1 & 1 & -1 & -1 & 0 & 0 & 0 & -2 & 2 & 1 & 1 & -1 & -1 & 0 & 0 \\
4 & 0 & 0 & 0 & -1 & -1 & -1 & -1 & -1 & -1 & 0 & 0 & 0 & -1 & -1 & -1 & -1 & -1 & -1
\end{array}\right)
$$

Finally, as it was explained before, $S_{k l}$ is a diagonal matrix. The entries of this diagonal matrix account for the relaxation times, $s_{i}$, of the different moments. In particular, in the D3Q19 scheme this reads:

$$
S_{k k}=\left(s_{0}, s_{1}, s_{2}, s_{3}, s_{4}, s_{5}, s_{6}, s_{7}, s_{8}, s_{9}, s_{10}, s_{11}, s_{12}, s_{13}, s_{14}, s_{15}, s_{16}, s_{17}, s_{18}\right)
$$

In the case of MRT collision model the hydrodynamic quantities (density and momentum) can be imposed with any value of $s_{0}, s_{1}, s_{2}$ and $s_{3}$, provided $\rho=$ $\rho^{e q}$ and $\mathbf{u}=\mathbf{u}^{e q}$. In what follows, they are set to zero.

Commonly in the literature, the rest of the relaxation times are set as the d'Humières' parameters [41]. To sum up, the d'Humières' diagonal matrix of 
the collision matrix of the MRT collision operators reads:

$$
S_{i i}=\left(0,0,0,0, s_{4}, s_{5}, s_{6}, s_{7}, s_{8}, s_{9}, 1.2,1.2,1.2,1.98,1.98,1.98,1.4,1.4,1.4\right) .
$$

As for the BGK collision operator, the Chapman-Enskog expansion establishes the relation between the relaxation times and the kinematic shear and bulk viscosities of the macroscopic fluid [13]. In the MRT approach the kinematic viscosity, $\nu$, and the bulk viscosity, $\eta$, are related to the relaxations parameters $\left(s_{4}, s_{5}, s_{6}, s_{7}, s_{8}, s_{9}\right)$ associated to the second order moments. As a result, this approach removes some of the constraints of the BGK collision operator. In particular, the viscosities read:

$$
\nu=c_{s}^{2}\left(\frac{1}{s_{i}}-\frac{\Delta t}{2}\right) \text { with } i=4, \ldots, 8, \eta=\frac{2}{D} c_{s}^{2}\left(\frac{1}{s_{9}}-\frac{\Delta t}{2}\right) .
$$

It is important to notice that, having identified the relations between the consequently the bulk viscosity is fixed to $6.7 \cdot 10^{-4} \frac{\mathrm{kg}}{\mathrm{ms}}$. That means a value of $\tau=0.503$ for the BGK collision operator and values $s_{4-9}=1.988$ for the MRT 
collision operators. The rest of relaxation times are fixed to the values proposed

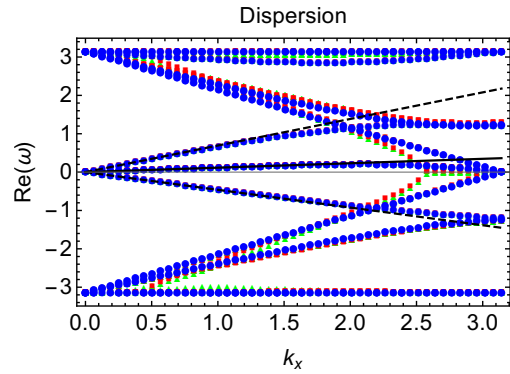

(a)

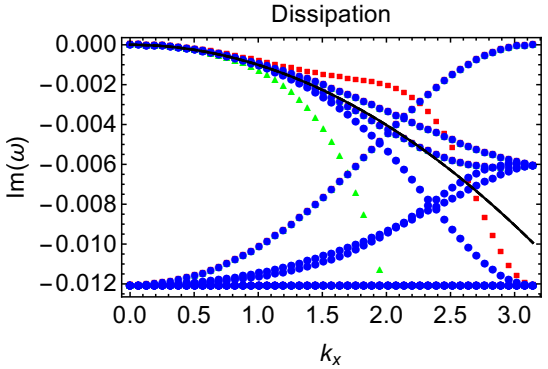

(b)

Figure B.7: Dispersion (a) and dissipation (b) for D3Q19 with BGK: •, d'Humières' MRT-RM: $\mathbf{\square}$, d'Humières' MRT-CM: $\Delta$ and theoretical modes (shear mode: - , and acoustic modes: - - ) at Ma $=0.2$ with $\nu=10^{-3} \frac{\mathrm{kg}}{\mathrm{ms}}$ (Note that the dissipation theoretical modes are overlapped).

Figure 7(b) shows dissipation where the theoretical modes (shear and acoustic) overlap, which is due the contribution of the kinematic and bulk viscosities in Eq. 8. Also as expected, the modes have a similar behaviour as in D2Q9 [16] regarding the dissipation of the shear mode. The MRT-CM approaches presents a higher dissipation rate at high wavenumbers.

Besides, the MRT-RM seems more unstable compared with the D2Q9 lattice scheme at the same fluid conditions (see Figure 3(b) in [16]). These differences between D2Q9 and D3Q19 lattice schemes could come from the different values used from the literature for the free parameters, Lallemand's [13] and d'Humieres' [41] values for D2Q9 and D3Q19 respectively. This assumption is clarified in the Appendix C. 


\section{Appendix C. Comparison between Lallemand's and d'Humières' parameters:}

As mentioned before, initially Lallemand and Luo [13] proposed values for the free parameters of the MRT collision operator for the D2Q9 lattice scheme. Later, d'Humières' [41] suggested different values for the D3Q19 MRT relaxation times. Both set of parameters were obtained through an optimization process based on linear stability analysis.

In this section, both Lallemand's and d'Humières' parameters are compared for the D3Q19. So, taking into account the geometrical relation between the D2Q9 and D3Q19 lattice schemes, the Lallemand's parameters for the D3Q19 discretization are:

$$
S_{i i}=\left(0,0,0,0, s_{4}, s_{5}, s_{6}, s_{7}, s_{8}, s_{9}, 1.9,1.9,1.9,1.9,1.9,1.9,1.54,1.54,1.54\right) .
$$

Figure C.8 shows the dispersion and dissipation for D3Q19 with Lallemand's parameters with $\nu=10^{-3} \frac{\mathrm{kg}}{\mathrm{ms}}$ and $\eta=6.7 \cdot 10^{-4} \frac{\mathrm{kg}}{\mathrm{ms}}\left(s_{4-9}=1.9988\right)$ at $\mathrm{Ma}=0.2$. Notice how the shear mode dissipation is identical compared with the D2Q9 lattice scheme (see Figure 3(b) in [16] ), which uses the Lallemand's parameters (Eq. C.1). These plots confirm the assumption done previously.

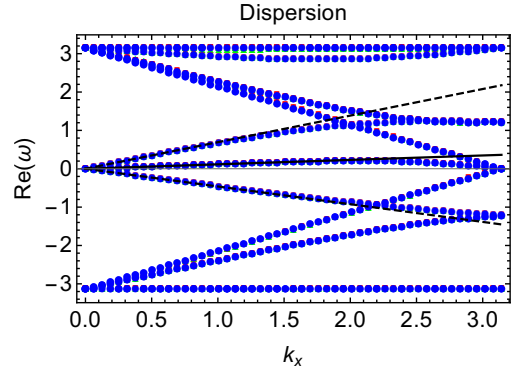

(a)

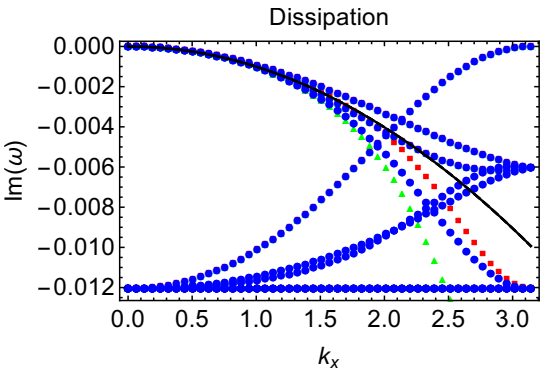

(b)

Figure C.8: Dispersion (a) and dissipation (b) for with D3Q19 BGK: •, Lallemand's MRT-RM: $\mathbf{\square}$, Lallemand's MRT-CM: $\Delta$ and theoretical modes (shear mode: - , and acoustic modes: - - ) with $\nu=10^{-3} \frac{\mathrm{kg}}{\mathrm{ms}}$ and $\eta=6.7 \cdot 10^{-4} \frac{\mathrm{kg}}{\mathrm{ms}}\left(s_{4-9}=1.9988\right)$ at $\mathrm{Ma}=0.2$ (Note that the dissipation theoretical modes are overlapped). 
Figure C.9 compares both Lallemand's and d'Humieres' parameters for the MRT-CM collision operator. Note how the dissipation of the shear mode is higher with d'Humières', because the second order moments are related with the shear mode dissipation. In this work, d'Humières' values are used due to the higher dissipation at high wavenumbers. This will prove advantageous to dissipate under-resolved waves.

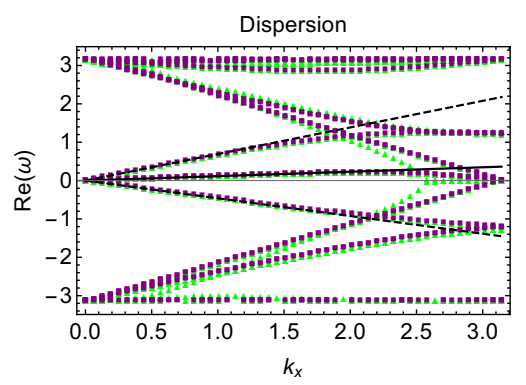

(a)

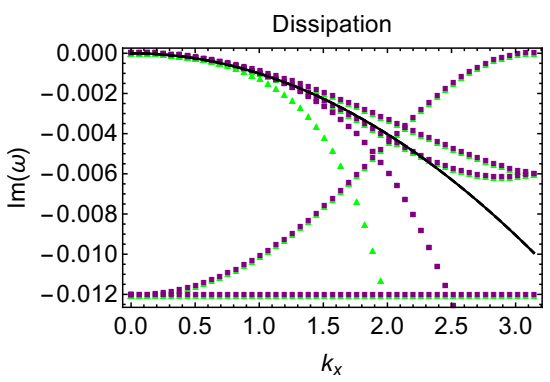

(b)

Figure C.9: Dispersion (a) and dissipation (b) for D3Q19 with Lallemand's MRT-CM: (ロ), d'Humières' MRT-CM: $(\Delta)$, and theoretical modes (shear mode, $\mathbf{C}$, and acoustic mode, - - ) with $\nu=10^{-3} \frac{\mathrm{kg}}{\mathrm{ms}}$ and $\eta=6.7 \cdot 10^{-4} \frac{\mathrm{kg}}{\mathrm{ms}}\left(s_{4-9}=1.994\right)$ at $\mathrm{Ma}=0.2$ (Note that the dissipation theoretical modes are overlapped). 


\section{References}

[1] S. Succi, The lattice Boltzmann equation, For Fluid Dynamics and Beyond (2001).

[2] H. Xu, P. Sagaut, Optimal low-dispersion low-dissipation LBM schemes for computational aeroacoustics, Journal of Computational Physics 230 (13) (2011) 5353-5382.

[3] R. Brionnaud, M. Chávez-Modena, G. Trapani, D. M. Holman, Direct noise computation with a lattice-Boltzmann method and application to industrial test cases, in: 22nd AIAA/CEAS Aeroacoustics Conference, 2016, p. 2969.

[4] D. Chatterjee, S. Chakraborty, A hybrid lattice Boltzmann model for solidliquid phase transition in presence of fluid flow, Physics Letters A 351 (4-5) (2006) 359-367.

[5] Y. Gan, A. Xu, G. Zhang, S. Succi, Discrete Boltzmann modeling of multiphase flows: hydrodynamic and thermodynamic non-equilibrium effects, Soft Matter 11 (26) (2015) 5336-5345.

[6] N. S. Martys, H. Chen, Simulation of multicomponent fluids in complex three-dimensional geometries by the lattice Boltzmann method, Physical review E 53 (1) (1996) 743.

[7] Y. H. Qian, D. D'Humières, P. Lallemand, Lattice BGK models for NavierStokes equation, EPL (Europhysics Letters) 17 (1992) 479.

[8] F. J. Higuera, J. Jiménez, Boltzmann approach to lattice gas simulations, EPL (Europhysics Letters) 9 (1989) 663.

[9] C. Coreixas, B. Chopard, J. Latt, Comprehensive comparison of collision models in the lattice boltzmann framework: Theoretical investigations, arXiv preprint arXiv:1904.12948 (2019). 
[10] P. L. Bhatnagar, E. P. Gross, M. Krook, A model for collision processes in gases. I. small amplitude processes in charged and neutral one-component systems, Physical review 94 (3) (1954) 511.

[11] Z. Guo, C. Zheng, T. Zhao, A lattice BGK scheme with general propagation, Journal of Scientific Computing 16 (4) (2001) 569-585.

[12] D. d'Humieres, Generalized lattice-Boltzmann equations, Progress in Astronautics and Aeronautics 159 (1994) 450-450.

[13] P. Lallemand, L.-S. Luo, Theory of the lattice Boltzmann method: Dispersion, dissipation, isotropy, galilean invariance, and stability, Physical Review E 61 (6) (2000) 6546.

[14] M. Geier, A. Greiner, J. G. Korvink, Cascaded digital lattice Boltzmann automata for high Reynolds number flow, Physical Review E 73 (6) (2006) 066705.

[15] M. Geier, A. Greiner, J. Korvink, A factorized central moment lattice Boltzmann method, The European Physical Journal Special Topics 171 (1) (2009) 55-61.

[16] M. Chávez-Modena, E. Ferrer, G. Rubio, Improving the stability of multiple-relaxation lattice Boltzmann methods with central moments, Computers \& Fluids (2018).

[17] J. G. Charney, R. Fjörtoft, J. v. Neumann, Numerical integration of the barotropic vorticity equation, Tellus 2 (4) (1950) 237-254.

[18] J. D. Sterling, S. Chen, Stability analysis of lattice Boltzmann methods, Journal of Computational Physics 123 (1) (1996) 196-206.

[19] D. Siebert, L. Hegele Jr, P. Philippi, Lattice Boltzmann equation linear stability analysis: thermal and athermal models, Physical Review E 77 (2) (2008) 026707. 
[20] O. Malaspinas, Increasing stability and accuracy of the lattice Boltzmann scheme: recursivity and regularization, arXiv preprint arXiv:1505.06900 (2015).

[21] K. K. Mattila, P. C. Philippi, L. A. Hegele Jr, High-order regularization in lattice-Boltzmann equations, Physics of Fluids 29 (4) (2017) 046103.

[22] C. Coreixas, G. Wissocq, G. Puigt, J.-F. Boussuge, P. Sagaut, Recursive regularization step for high-order lattice Boltzmann methods, Physical Review E 96 (3) (2017) 033306.

[23] S. Marié, D. Ricot, P. Sagaut, Comparison between lattice Boltzmann method and Navier-Stokes high order schemes for computational aeroacoustics, Journal of Computational Physics 228 (4) (2009) 10561070.

[24] F. Dubois, T. Fevrier, B. Graille, On the stability of a relative velocity lattice Boltzmann scheme for compressible Navier-Stokes equations, Comptes Rendus Mécanique 343 (10) (2015) 599-610.

[25] G. Wissocq, P. Sagaut, J.-F. Boussuge, An extended spectral analysis of the lattice Boltzmann method: modal interactions and stability issues, Journal of Computational Physics 380 (2019) 311-333.

[26] S. Chapman, T. G. Cowling, The mathematical theory of non-uniform gases: an account of the kinetic theory of viscosity, thermal conduction and diffusion in gases, Cambridge university press, 1970.

[27] H. Xu, O. Malaspinas, P. Sagaut, Sensitivity analysis and determination of free relaxation parameters for the weakly-compressible MRT-LBM schemes, Journal of Computational Physics 231 (21) (2012) 7335-7367.

[28] R. Moura, S. Sherwin, J. Peiró, Linear dispersion-diffusion analysis and its application to under-resolved turbulence simulations using Discontinuous Galerkin spectral/hp methods, Journal of Computational Physics 298 (2015) 695-710. 
[29] G. I. Taylor, A. E. Green, Mechanism of the production of small eddies from large ones, Proc. R. Soc. Lond. A 158 (895) (1937) 499-521.

[37] L. Landau, E. Lifshitz, Fluid mechanics: Landau and Lifshitz: course of theoretical physics, Vol. 6, Elsevier, 2013.

[38] G. Gassner, D. A. Kopriva, A comparison of the dispersion and dissipation errors of Gauss and Gauss-Lobatto discontinuous Galerkin spectral 

2560-2579.

[39] J. Manzanero, G. Rubio, E. Ferrer, E. Valero, Dispersion-diffusion analysis for advection problems with non-constant coefficients: applications to Discontinuous Galerkin formulations., SIAM Journal of Scientific Computing 40 (2) (2018) A747-A768.

[40] J. Manzanero, E. Ferrer, G. Rubio, E. Valero, On the role of numerical dissipation in stabilising under-resolved turbulent simulations using discontinuous Galerkin methods, arXiv preprint arXiv:1805.10519 (2018).

555 [41] D. d'Humières, Multiple-relaxation-time lattice Boltzmann models in three dimensions, Philosophical Transactions of the Royal Society of London. Series A: Mathematical, Physical and Engineering Sciences 360 (1792) (2002) 437-451. 